\title{
Wide-field endoscopic submucosal dissection for whole-antral and whole-incisura gastric multifocal dysplasia
}

\section{Georgios Mavrogenis ${ }^{a}$, Fateh Bazerbachib, Nikolaos Kokolasc, Loukas Kaklamanis ${ }^{d}$}

Mediterraneo Hospital, Athens Greece; Massachusetts General Hospital, Boston, USA, Onassis Cardiac Surgery Center, Athens, Greece

Gastric endoscopic submucosal dissection (ESD) is applied for early gastric cancer $(<3 \mathrm{~cm})$ or isolated visible lesions with dysplasia [1]. In this report, we describe whole-antral and whole-incisura ESD applications for the resection of diffuse enteric metaplasia with multifocal dysplasia.

In case 1, an 80-year-old male underwent EGD for dyspepsia, and was found to have diffuse irregularity of the gastric incisura (Paris IIa+IIb) creeping towards the pylorus and the anterior and posterior wall of the antrum (Fig. 1). Low-grade dysplasia was detected in mucosal biopsies. ESD with dynamic clip and band countertraction [2] was performed and the abnormality was excised. The specimen measured $7.6 \mathrm{~cm} \times 7 \mathrm{~cm}$ in size, and histopathology demonstrated multifocal low and high-grade dysplasia with negative margins.

In case 2, a 60-year-old male was found to have a low grade dysplastic lesion at the lesser curvature, extending towards the incisura and proximal antrum. Multifocal clip and band ESD was applied [2], and the lesion was excised en bloc (Fig. 2). The specimen, measuring $7 \mathrm{~cm} \times 7 \mathrm{~cm}$, harbored multifocal low-grade dysplasia with negative margins. Both patients were hospitalized for 24 hours and had an uneventful recovery.

These two cases illustrate the feasibility of wide-field wholeantral and whole-incisura ESD for extensive dysplastic areas. Despite the necessity for technical expertise, this technique can be facilitated by the application of ancillary techniques, such as dynamic and multifocal countertraction, as previously described by our team [2,3]. En-bloc resection offers obvious advantages in terms of decreasing recurrence risk, and obviating repeat interventions.

\footnotetext{
Department of a Gastroenterology, Mediterraneo Hospital, Athens, Greece (Georgios Mavrogenis); 'bastroenterology, Massachusetts General Hospital, Boston, USA (Fateh Bazerbachi); 'Anesthesiology, Mediterraneo Hospital, Athens, Greece (Nikolaos Kokolas); ${ }^{\text {PPathology, }}$ Onassis Cardiac Surgery Center, Athens, Greece (Loukas Kaklamanis)

\section{Conflict of Interest: None}

Correspondence to: Georgios Mavrogenis, MD, Director of Hybrid Interventional Endoscopy, Department of Gastroenterology, Mediterraneo Hospital, Ilias 12, 16675 Athens, Greece, e-mail: mavrogenis@gmail.com
}

Received 1 November 2020; accepted 20 November 2020 published online 27 January 2021

DOI: https://doi.org/10.20524/aog.2021.0588

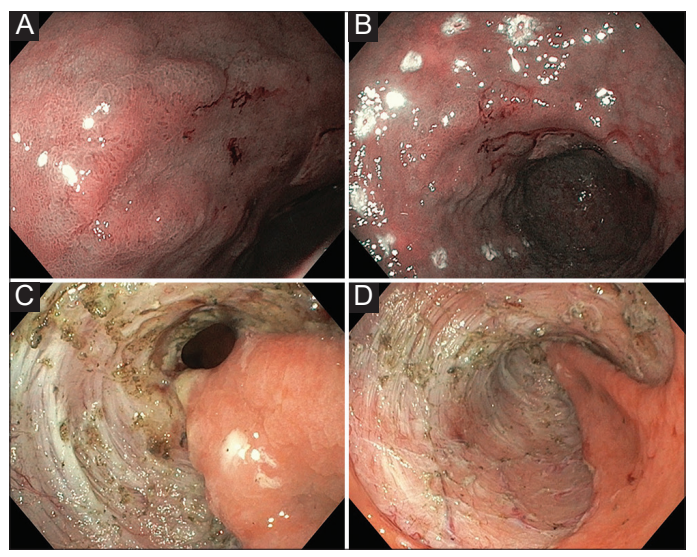

Figure 1 Case 1. (A) Narrow-band imaging of a Paris IIa+IIb dysplastic lesion of the incisura. (B) Marking of the left part of the lesion. (C, D) Extensive resection of the mucosa covering the antrum and incisura

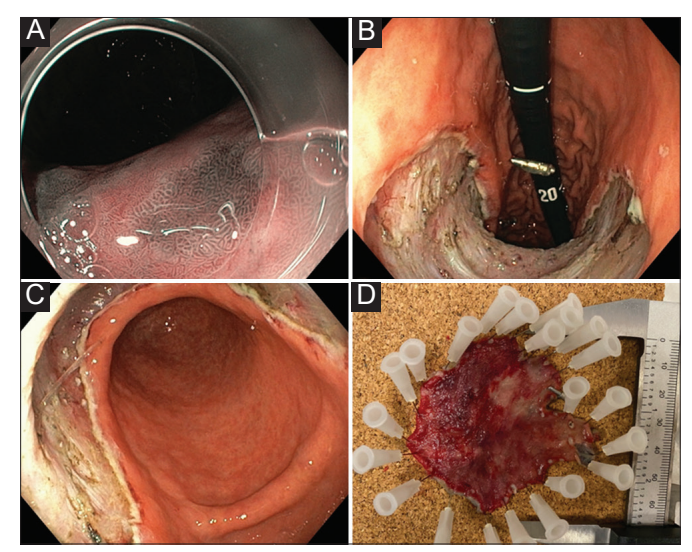

Figure 2 Case 2. (A) Multifocal dysplasia of the incisura (Paris IIb). $(\mathrm{B}, \mathrm{C})$ Extensive mucosal resection of the lesser curvature. (D) Specimen

\section{References}

1. Pimentel-Nunes P, Dinis-Ribeiro M, Ponchon T, et al. Endoscopic submucosal dissection: European Society of Gastrointestinal Endoscopy (ESGE) Guideline. Endoscopy 2015;47:829-854.

2. Mavrogenis G, Bazerbachi F, Tsevgas I, Zachariadis D. Dynamic and multifocal clip and band countertraction for endoscopic submucosal dissection. VideoGIE 2020;5:451-454.

3. Mavrogenis G, Mavrogenis I, Anastasiadis S, Bazerbachi F. Underwater endoscopic submucosal dissection in saline solution with rubber-band countertraction for a cecal polyp extending into a diverticulum. Ann Gastroenterol 2019;32:527. 